\title{
The speciation of copper (II)/ethylenediamine/oxalate system by evolving factor analysis
}

\begin{abstract}
Shouxin Ren and Ling Gao
Department of Chemistry, Inner Mongolian University, Huhehot 010021, Inner Mongolia, China

Principal component analysis and evolving factor analysis were applied to the study of the speciation of $C u(I I) /$ ethylenediamine/ oxalate. Two programs were designed, based on mathematical algorithms. Error functions were calculated for evaluating the number of species. Submatrix analysis plots were constructed to estimate the species present in the system. The method should prove useful in studies of complex systems in environmental samples.
\end{abstract}

\section{Introduction}

Information about the speciation of metals in natural water is important in studies of the toxicity of metals for aquatic organisms. The study of speciation also contributes to the understanding of trace metal transport in the water environment. Biological effects on aquatic organisms and geochemical behaviour both depend greatly on the species of the element so the study of speciation has been one of the most important areas of environmental and analytical chemistry in recent years.

Studies of multiple equilibria in solution have solved a number of problems in speciation. Traditional approaches use least squares methods based on a chemical model and on compliance with the law of mass action [1]. A variety of instrumental techniques have been used for the determination of the number, nature and stabilities of multiple equilibria systems. Spectrophotometry has considerable advantages over the other techniques because of its simplicity. Gampp et al. [2] proposed a model-free method derived from principal component analysis (PGA): evolving factor analysis (EFA). EFA is a powerful method for analysing multivariate data with an intrinsic order produced by many modern hyphenated instruments, such as speciation in multiple equilibria systems using spectrophotometric titration. Data can be listed, according to its $\mathrm{pH}$, from low to high. The first columns may contain the data measured at low $\mathrm{pH}$ values and the last columns those obtained at high $\mathrm{pH}$ values. The method is based upon repetitive eigenvalue analysis of a set of data matrices obtained during the evolutionary process. Eigenanalysis is performed on a complete series of matrices, which are constructed by successively adding spectra to the previous matrix during the evolutionary process. As new absorbing species evolve, the eigenvalues of the abstract factors increase by an order of magnitude. The logarithmic eigenvalues are plotted as a function of the progessing titration. Every submatrix is calculated by PCA in turn.

\section{Experimental}

\section{Instruments and apparatus}

The Shimadzu UV-265 and UV-120 spectrophotometers were used for all experiments; a GW $286 \mathrm{EX} / 16$ microcomputer with a maths coprocessor was used for the calculations; and a Mettler DL21 titrator was used for standardization of standard solutions.

\section{Reagents}

All reagents were of analytical reagent grade. Doubly distilled and de-ionized water were used. Standard solutions of copper(II) nitrate, ethylenediamine and potassium oxalate were prepared and standardized according to generally accepted procedures.

\section{Spectrophotometric titration}

Solutions which contain $\mathrm{Cu}(\mathrm{II})$ ion and one or two of the ligands were titrated with a base at a constant ionic strength $(0.5 \mathrm{M})$ and temperature $\left(25^{\circ} \mathrm{C}\right)$. After each addition of titrant and equilibration, the $\mathrm{pH}$ was measured. The absorbance of spectra of each solution was measured at a different $\mathrm{pH}$, with a wavelength range from $480 \mathrm{~nm}$ to $820 \mathrm{~nm}$, in $20 \mathrm{~nm}$ intervals. An experimental data. matrix, $D$, was built up from these data.

\section{Computer programs and their algorithm}

The steps of the algorithm were as follows:

(1) Building the data matrix $D(\mathcal{N}, M)$. Matrix $D$ contains the spectra at $\mathcal{N}$ wavelengths of the $M$ mixtures obtained at the successive titration points of the spectrometric titration.

(2) Factor analysis of the experimental data matrix $D$. The variance-covariance matrix $A\left(D^{\mathrm{T}} D\right)$ can be subjected to single value decomposition for calculation of the eigenvalues and eigenvectors. The number of species is estimated by several criteria based on the theory of error in factor analysis [3].

(3) Evolving factor analysis. The fundamental idea of EFA is to follow the evolution of the rank of the data matrix $D$ as a function of the ordered variable, for example $\mathrm{pH}$ in a spectrophotometric titration. This is done by PCA on an increasing data matrix. The logarithm of the singular values corresponding to the principal components determined by PCA versus increasing and decreasing $\mathrm{pH}$, are plotted in the same graph. 
Table 1. Summary of the conditions for the experimental determinations.

\begin{tabular}{|c|c|c|c|c|c|}
\hline \multirow[t]{2}{*}{ System } & \multicolumn{3}{|c|}{ Molar concentrations } & \multirow{2}{*}{$\begin{array}{l}\text { Wavelength } \\
\text { range } \\
(\mathrm{nm})\end{array}$} & \multirow{2}{*}{$\underset{\text { range }}{\mathrm{pH}}$} \\
\hline & $\mathrm{Cu}(\mathrm{II})$ & ox & en & & \\
\hline $\mathrm{Cu}(\mathrm{II}) / \mathrm{ox}$ & $0 \cdot 0100$ & $0 \cdot 1000$ & - & $480-820$ & $3 \cdot 5-10 \cdot 0$ \\
\hline $\mathrm{Cu}(\mathrm{II}) / \mathrm{en}$ & $0 \cdot 0100$ & - & $0 \cdot 1000$ & $480-820$ & $4 \cdot 0-11 \cdot 0$ \\
\hline $\mathrm{Cu}(\mathrm{II}) / \mathrm{ox} / \mathrm{cn}$ & $0 \cdot 0100$ & $0 \cdot 1000$ & $0 \cdot 1000$ & $480-820$ & $3 \cdot 5-11 \cdot 0$ \\
\hline
\end{tabular}

Table 2. Principal component analysis applied to $C u(I I) / o x /$ en system.

\begin{tabular}{|c|c|c|c|c|c|c|c|c|c|}
\hline$n$ & EV & $\mathrm{RE}$ & IE & $\mathrm{XE}$ & IND & ER & Frac & REV & \multirow{2}{*}{$\begin{array}{l}\text { REV } \\
\text { ratio }\end{array}$} \\
\hline \multicolumn{9}{|c|}{$\times 10^{3}$} & \\
\hline 1 & $6 \cdot 3025$ & $0 \cdot 0675$ & $0 \cdot 0169$ & $0 \cdot 0654$ & $0 \cdot 3002$ & $5 \cdot 5629$ & $0 \cdot 84419$ & $0 \cdot 02317093$ & 4.92 \\
\hline 2 & $1 \cdot 1330$ & $0 \cdot 0113$ & $0 \cdot 0040$ & $0 \cdot 0106$ & $0 \cdot 0576$ & 38.4695 & $0 \cdot 15175$ & $0 \cdot 00472065$ & 33.66 \\
\hline 3 & $0 \cdot 0295$ & $0 \cdot 0020$ & $0 \cdot 0009$ & $0 \cdot 0028$ & $0 \cdot 0116$ & $83 \cdot 8337$ & $0 \cdot 00395$ & $0 \cdot 00014024$ & $72 \cdot 66$ \\
\hline 4 & $0 \cdot 0004$ & $0 \cdot 0016$ & $0 \cdot 0008$ & $0 \cdot 0014$ & $0 \cdot 0109$ & $2 \cdot 0273$ & $0 \cdot 00005$ & $0 \cdot 00000193$ & $\begin{array}{r}12.00 \\
1.74\end{array}$ \\
\hline 5 & $0 \cdot 0002$ & $0 \cdot 0013$ & $0 \cdot 0007$ & $0 \cdot 0011$ & $0 \cdot 0109$ & $1 \cdot 4798$ & $0 \cdot 00002$ & $0 \cdot 00000111$ & 1.25 \\
\hline 6 & $0 \cdot 0001$ & $0 \cdot 0011$ & $0 \cdot 0007$ & $0 \cdot 0009$ & $0 \cdot 0111$ & $1 \cdot 7345$ & $0 \cdot 00002$ & $0 \cdot 00000088$ & $\begin{array}{l}1.20 \\
1.45\end{array}$ \\
\hline 7 & $0 \cdot 0001$ & $0 \cdot 0010$ & $0 \cdot 0006$ & $0 \cdot 0007$ & $0 \cdot 0119$ & $1 \cdot 4184$ & $0 \cdot 00000$ & 0.00000061 & \\
\hline 8 & $0 \cdot 0000$ & $0 \cdot 0008$ & $0 \cdot 0006$ & $0 \cdot 0006$ & $0 \cdot 0131$ & $1 \cdot 4847$ & $0 \cdot 00000$ & $0 \cdot 00000052$ & $\begin{array}{l}1.10 \\
1.19\end{array}$ \\
\hline 9 & $0 \cdot 0000$ & $0 \cdot 0007$ & $0 \cdot 0005$ & $0 \cdot 0005$ & $0 \cdot 0148$ & $1 \cdot 2979$ & $0 \cdot 00000$ & $0 \cdot 00000045$ & $\begin{array}{l}1.19 \\
1.01\end{array}$ \\
\hline 10 & $0 \cdot 0000$ & $0 \cdot 0006$ & $0 \cdot 0005$ & $0 \cdot 0004$ & $0 \cdot 0170$ & $1 \cdot 8138$ & 0.00000 & 0.0000004 .4 & $\begin{array}{l}1.01 \\
1.36\end{array}$ \\
\hline 11 & $0 \cdot 0000$ & $0 \cdot 0005$ & $0 \cdot 0004$ & $0 \cdot 0003$ & $0 \cdot 0215$ & $1 \cdot 2982$ & $0 \cdot 00000$ & $0 \cdot 00000032$ & $\begin{array}{l}1.30 \\
0.93\end{array}$ \\
\hline 12 & $0 \cdot 0000$ & $0 \cdot 0005$ & $0 \cdot 0004$ & $0 \cdot 0002$ & $0 \cdot 0284$ & $1 \cdot 3170$ & $0 \cdot 00000$ & $0 \cdot 00000035$ & $\begin{array}{l}0.93 \\
0.88\end{array}$ \\
\hline 13 & $0 \cdot 0000$ & $0 \cdot 0003$ & $0 \cdot 0003$ & $0 \cdot 0001$ & $0 \cdot 0384$ & 1.9767 & $0 \cdot 00000$ & $0 \cdot 00000040$ & 1.19 \\
\hline 14 & $0 \cdot 0000$ & $0 \cdot 0002$ & $0 \cdot 0002$ & $0 \cdot 0001$ & $0 \cdot 0614$ & $2 \cdot 0204$ & $0 \cdot 00000$ & 0.00000034 & $\begin{array}{l}1.19 \\
1.01\end{array}$ \\
\hline 15 & $0 \cdot 0000$ & $0 \cdot 0001$ & $0 \cdot 0001$ & $0 \cdot 0000$ & $0 \cdot 0582$ & $34 \cdot 6303$ & $0 \cdot 00000$ & $0 \cdot 00000034$ & 101 \\
\hline 16 & $0 \cdot 0000$ & & & & & & $0 \cdot 00000$ & 0.00000002 & 1104 \\
\hline
\end{tabular}

The plots perform the submatrix analysis of the titration data. Two programs, SPGRAFA and SPGREFA, which were based on the algorithm, were designed to perform the calculation.

\section{Results and discussion}

Three different systems were used in this work:

(1) $\mathrm{Cu}($ II $) /$ oxalate $($ ox $)$.

(2) $\mathrm{Cu}($ II $) /$ ethylenediamine (en).

(3) $\mathrm{Cu}(\mathrm{II}) / \mathrm{ox} / \mathrm{en}$.

The initial conditions used in the spectrophotometric titrations are shown in table 1 . High ratios of ligand to copper ion were used in order to avoid copper hydroxide precipitation at neutral $\mathrm{pH}$.

\section{Estimating the number of species by factor analysis}

Nine criteria were used to estimate the number of species. Table 2 shows the principal component analysis applied to the $\mathrm{Cu}(\mathrm{II}) / \mathrm{ox} / \mathrm{en}$. When six components were considered, the real error or residual standard deviation function, RE, had a value of 0.0011 ; the imbedded error function, IE, had a value of 0.007; and the extracted error, $\mathrm{XE}$, had a value of $0 \cdot 0009$. In general, the RE function with values larger than 0.001 is for the expected number of components, thus a range somewhat above this value is the boundary between wanted and unwanted information. Malinowski's empirical factor function, IND, reached a minimum betweeen 4 and 6 ; the function increased rapidly after $\mathcal{N}=6$. A maximum of the eigenvalue ratio function, ER, appeared at 6 [4]. In 1987, Malinowski proposed calculation of reduced eigenvalues, REV [5]; REV values are calculated according to the equation:

$$
\mathrm{REV}_{j}=\lambda_{j} /(l-j+1)(k-j+1)
$$

where $l$ and $k$ are the number of rows and columns in matrix $A$, assuming that $l$ is greater than $k ; \mathrm{REV}_{j}$ is the $j$ th reduced eigenvalue; and $j$ is the order number. The magnitude of REV decreased rapidly in the system until stabilizing at $\mathcal{N}=6$. As recommended by Gemperline and Hamilton [6], the REV ratio can be used to estimate the number of significant components in data matrix. Starting with the least significant eigenvalues, $j=k, \mathrm{REV}_{j}$ is calculated. The ratio $\mathrm{Y}_{j-1}$ is obtained according to the following equation:

$$
r_{j-1}=\mathrm{REV}_{j-1} / \mathrm{REV}_{j}
$$

When $\lambda_{j}$ and $\lambda_{j-1}$ are error eigenvalues, then the ratio $Y_{j-1}$ is approximately equal to one. If the REV ratios from $r_{5}$ to $r_{14}$ are approximately equal to one, then the eigenvalues from $\mathcal{N}=5$ are all the error eigenvalues. The newer indicator function $\mathrm{Frac}_{i}$ suggested by Malinowski 
is given by the equation:

$$
\operatorname{Frac}_{i}=\lambda_{i} / \sum_{j=1}^{k} \lambda_{j}
$$

where $\lambda_{i}$ is the $i$ th eigenvalue, and the sum is over that eigenvalue and all the remaining eigenvalues. The appropriate number of components is one less than that giving the minimum $\mathrm{Frac}_{i}$ value. In the system, the Frac had a minimum at $\mathcal{N}=7$, after 7 the change in Frac was quite small, suggesting that 6 was the optimum component number. From these criteria, it was concluded that six absorbing species were present.

SPGRAFA was also used to deduce the number of components for $\mathrm{Cu}(\mathrm{II}) / \mathrm{en}$ and $\mathrm{Cu}(\mathrm{II}) /$ ox systems. For the $\mathrm{Cu}(\mathrm{II}) / \mathrm{en}$ system, the results indicated that no appreciable decreases in $\mathrm{RE}, \mathrm{IE}$ and $\mathrm{XE}$ values were observed from the number of components $\mathcal{N}=3$ to 14 . A maximum of the ER function appeared at $\mathcal{N}=3$. Although the IND function was not at a minimum at $\mathcal{N}=3$, at $\mathcal{N}=4$ the largest difference for this function appeared between $\mathcal{N}=2$ and 3 , which indicated that three absorbing components were present. The magnitude of the first three eigenvalues were larger that those of $\mathcal{N}=4-14$. The magnitude of the eigenvalue represents the importance of the contribution that each eigenvector makes to fitting the real data. Therefore, three species were formed in the system. Chemical reasoning may deduce the formation of $\mathrm{Cu}(\mathrm{II}),[\mathrm{Cu}(\mathrm{en})]^{2+}$ and $\left[\mathrm{Cu}(\mathrm{en})_{2}\right]^{2+}$. Similar results were obtained for the $\mathrm{Cu}(\mathrm{II}) / \mathrm{ox}$ system with SPGRAFA. Three absorbing species were present in the mixture solution; these could have been $\mathrm{Cu}(\mathrm{II}),[\mathrm{Cu}(\mathrm{ox})]$ and $\left[\mathrm{Cu}(\mathrm{ox})_{2}\right]^{2+}$. There is an inherent difficulty in trying to determine the exact number of species in complicated systems simply by observing the error functions; submatrix analysis plots at varying $\mathrm{pHs}$ are more useful for this.

\section{Evolving factor analysis of $\mathrm{Cu}(I I) /$ oxalate/ethylenediamine system}

As the first step, PGA is performed on the submatrix $D_{i}$ to calculate the singular value (SV) of the first $i=1,2-16$. The SVs obtained from backward EFA on the last $i=1,2-16$ spectra were calculated in a similar way.

It was assumed that the least SVs were zero and the results of the forward and backward EFAs are given in tables 3 and 4. The logarithms of the SVs are more suitable for graphical representation; the SVs from the forward and backward EFA for the $\mathrm{Cu}(\mathrm{II}) / \mathrm{ox} / \mathrm{en}$ system are plotted in figure 1. The number of species is determined visually, while the SVs caused by an absorbing substance show a sharp increase as a function of $\mathrm{pH}$, which is due to the evolution of a species; those caused by error factor increase only very little. Figure 1 shows that six major components

Table 3. $\log (S V)$ of forward EFA.

\begin{tabular}{|c|c|c|c|c|c|c|c|c|}
\hline $\mathrm{pH}$ & $\mathrm{SV}_{1}$ & $\mathrm{SV}_{2}$ & $\mathrm{SV}_{3}$ & $\mathrm{SV}_{4}$ & $\mathrm{SV}_{5}$ & $\mathrm{SV}_{6}$ & $\mathrm{SV}_{7}$ & $\mathrm{SV}_{8}$ \\
\hline $4 \cdot 0$ & 0.5313 & 0.0064 & 0 & 0 & 0 & 0 & 0 & 0 \\
\hline $4 \cdot 5$ & $0 \cdot 6536$ & $0 \cdot 0075$ & $0 \cdot 0056$ & 0 & 0 & 0 & 0 & 0 \\
\hline $5 \cdot 0$ & $0 \cdot 7401$ & $0 \cdot 0251$ & $0 \cdot 0070$ & $0 \cdot 0050$ & 0 & 0 & 0 & 0 \\
\hline $5 \cdot 5$ & $0 \cdot 8157$ & $0 \cdot 0400$ & $0 \cdot 0086$ & $0 \cdot 0053$ & $0 \cdot 0017$ & 0 & 0 & 0 \\
\hline $6 \cdot 0$ & $0 \cdot 8655$ & $0 \cdot 1095$ & $0 \cdot 0134$ & $0 \cdot 0053$ & $0 \cdot 0022$ & $0 \cdot 0012$ & 0 & 0 \\
\hline $6 \cdot 5$ & $0 \cdot 8944$ & $0 \cdot 1925$ & 0.0310 & 0.0061 & 0.0031 & $0 \cdot 0022$ & 0.0012 & 0 \\
\hline $7 \cdot 5$ & $0 \cdot 9189$ & $0 \cdot 3541$ & 0.0700 & $0 \cdot 0066$ & 0.0045 & $0 \cdot 0038$ & 0.0029 & $0 \cdot 0020$ \\
\hline $8 \cdot 0$ & 0.9315 & $0 \cdot 4232$ & 0.0755 & $0 \cdot 0067$ & $0 \cdot 0045$ & $0 \cdot 0039$ & 0.0035 & $0 \cdot 0028$ \\
\hline $8 \cdot 5$ & 0.9440 & $0 \cdot 4707$ & $0 \cdot 0783$ & 0.0067 & $0 \cdot 0048$ & $0 \cdot 0041$ & $0 \cdot 0036$ & $0 \cdot 0028$ \\
\hline $9 \cdot 0$ & $0 \cdot 9570$ & 0.5086 & $0 \cdot 0800$ & $0 \cdot 0068$ & $0 \cdot 0051$ & $0 \cdot 0042$ & 0.0038 & $0 \cdot 0034$ \\
\hline 9.5 & $0 \cdot 9741$ & $0 \cdot 5454$ & $0 \cdot 0811$ & $0 \cdot 0068$ & $0 \cdot 0051$ & $0 \cdot 0043$ & $0 \cdot 0038$ & $0 \cdot 0034$ \\
\hline $10 \cdot 0$ & 0.9933 & 0.5777 & $0 \cdot 0821$ & $0 \cdot 0077$ & $0 \cdot 0057$ & $0 \cdot 0050$ & 0.0043 & $0 \cdot 0037$ \\
\hline $10 \cdot 5$ & $1 \cdot 0153$ & $0 \cdot 6017$ & $0 \cdot 0832$ & $0 \cdot 0078$ & 0.0057 & $0 \cdot 0050$ & $0 \cdot 0043$ & $0 \cdot 0038$ \\
\hline $\mathrm{pH}$ & $\mathrm{SV}_{9}$ & $\mathrm{SV}_{10}$ & $\mathrm{SV}_{11}$ & $\mathrm{SV}_{12}$ & $\mathrm{SV}_{13}$ & $\mathrm{SV}_{14}$ & $\mathrm{SV}_{15}$ & $\mathrm{SV}_{16}$ \\
\hline $4 \cdot 0$ & 0 & 0 & 0 & 0 & 0 & 0 & 0 & 0 \\
\hline $4 \cdot 5$ & 0 & 0 & 0 & 0 & 0 & 0 & 0 & 0 \\
\hline $5 \cdot 0$ & 0 & 0 & 0 & 0 & 0 & 0 & 0 & 0 \\
\hline $5 \cdot 5$ & 0 & 0 & 0 & 0 & 0 & 0 & 0 & 0 \\
\hline $6 \cdot 0$ & 0 & 0 & 0 & 0 & 0 & 0 & 0 & 0 \\
\hline $6 \cdot 5$ & 0 & 0 & 0 & 0 & 0 & 0 & 0 & 0 \\
\hline $7 \cdot 0$ & 0 & 0 & 0 & 0 & 0 & 0 & 0 & 0 \\
\hline $7 \cdot 5$ & $0 \cdot 0011$ & 0 & 0 & 0 & 0 & 0 & 0 & 0 \\
\hline $8 \cdot 0$ & $0 \cdot 0020$ & $0 \cdot 0009$ & 0 & 0 & 0 & 0 & 0 & 0 \\
\hline $8 \cdot 5$ & $0 \cdot 0020$ & 0.0012 & $0 \cdot 0009$ & 0 & 0 & 0 & 0 & 0 \\
\hline $9 \cdot 0$ & $0 \cdot 0027$ & $0 \cdot 0020$ & 0.0010 & 0.0007 & 0 & 0 & 0 & 0 \\
\hline $9 \cdot 5$ & $0 \cdot 0029$ & $0 \cdot 0027$ & 0.0014 & 0.0010 & 0.0007 & 0 & 0 & 0 \\
\hline $10 \cdot 0$ & $0 \cdot 0029$ & $0 \cdot 0028$ & 0.0015 & $0 \cdot 0012$ & 0.0010 & 0.0007 & 0 & 0 \\
\hline
\end{tabular}


Table 4. $\log (S V)$ of backward EFA.

\begin{tabular}{|c|c|c|c|c|c|c|c|c|}
\hline $\mathrm{pH}$ & $\mathrm{SV}_{1}$ & $\mathrm{SV}_{2}$ & $\mathrm{SV}_{3}$ & $\mathrm{SV}_{4}$ & $\mathrm{SV}_{5}$ & $\mathrm{SV}_{6}$ & $\mathrm{SV}_{7}$ & $\mathrm{SV}_{8}$ \\
\hline $3 \cdot 5$ & $1 \cdot 0414$ & 0.6227 & 0.0842 & 0.0081 & 0.0058 & $0 \cdot 0050$ & 0.0044 & $0 \cdot 0038$ \\
\hline $4 \cdot 0$ & 0.9910 & 0.5835 & 0.0793 & $0 \cdot 0081$ & 0.0055 & 0.0050 & $0 \cdot 0043$ & $0 \cdot 0035$ \\
\hline $4 \cdot 5$ & $0 \cdot 9530$ & $0 \cdot 5324$ & 0.0737 & $0 \cdot 0080$ & $0 \cdot 0052$ & $0 \cdot 0049$ & $0 \cdot 0043$ & $0 \cdot 0035$ \\
\hline $5 \cdot 0$ & 0.9163 & 0.4571 & $0 \cdot 0610$ & 0.0074 & 0.0051 & 0.0043 & 0.0035 & $0 \cdot 0034$ \\
\hline $5 \cdot 5$ & $0 \cdot 8889$ & $0 \cdot 3715$ & $0 \cdot 0530$ & $0 \cdot 0073$ & 0.0051 & $0 \cdot 0043$ & $0 \cdot 0034$ & 0.0034 \\
\hline $6 \cdot 0$ & $0 \cdot 8645$ & $0 \cdot 2538$ & $0 \cdot 0288$ & 0.0071 & $0 \cdot 0043$ & $0 \cdot 0038$ & $0 \cdot 0034$ & 0.0032 \\
\hline $6 \cdot 5$ & $0 \cdot 8360$ & $0 \cdot 1377$ & 0.0139 & 0.0061 & 0.0043 & 0.0038 & 0.0033 & $0 \cdot 0024$ \\
\hline $7 \cdot 0$ & $0 \cdot 7998$ & 0.0397 & 0.0139 & 0.0060 & 0.0042 & 0.0034 & 0.0026 & $0 \cdot 0018$ \\
\hline $7 \cdot 5$ & $0 \cdot 7580$ & $0 \cdot 0204$ & $0 \cdot 0086$ & $0 \cdot 0047$ & $0 \cdot 0038$ & $0 \cdot 0027$ & $0 \cdot 0019$ & 0.0011 \\
\hline $8 \cdot 0$ & $0 \cdot 7102$ & $0 \cdot 0204$ & $0 \cdot 0056$ & 0.0044 & $0 \cdot 0028$ & 0.0020 & 0.0012 & 0 \\
\hline $8 \cdot 5$ & 0.6532 & $0 \cdot 0182$ & $0 \cdot 0056$ & 0.0039 & 0.0025 & 0.0016 & 0 & 0 \\
\hline $9 \cdot 0$ & $0 \cdot 6003$ & $0 \cdot 0170$ & $0 \cdot 0054$ & $0 \cdot 0039$ & $0 \cdot 0017$ & 0 & 0 & 0 \\
\hline $9 \cdot 5$ & 0.5461 & $0 \cdot 0150$ & 0.0052 & 0.0018 & 0 & 0 & 0 & 0 \\
\hline $10 \cdot 0$ & 0.4757 & 0.0132 & 0.0024 & 0 & 0 & 0 & 0 & 0 \\
\hline $10 \cdot 5$ & $0 \cdot 3904$ & $0 \cdot 0038$ & 0 & 0 & 0 & 0 & 0 & 0 \\
\hline $\mathrm{pH}$ & $\mathrm{SV}_{9}$ & $\mathrm{SV}_{10}$ & $\mathrm{SV}_{11}$ & $\mathrm{SV}_{12}$ & $\mathrm{SV}_{13}$ & $\mathrm{SV}_{14}$ & $\mathrm{SV}_{15}$ & $\mathrm{SV}_{16}$ \\
\hline $3 \cdot 5$ & $0 \cdot 0035$ & $0 \cdot 0030$ & $0 \cdot 0023$ & 0.0015 & $0 \cdot 0012$ & 0.0011 & $0 \cdot 0007$ & 0 \\
\hline $4 \cdot 0$ & 0.0034 & 0.0029 & 0.0022 & 0.0015 & 0.0012 & 0.0008 & $0 \cdot 0006$ & 0 \\
\hline $4 \cdot 5$ & $0 \cdot 0034$ & $0 \cdot 0029$ & 0.0022 & 0.0014 & $0 \cdot 0011$ & $0 \cdot 0008$ & 0 & 0 \\
\hline $5 \cdot 0$ & $0 \cdot 0030$ & $0 \cdot 0022$ & $0 \cdot 0014$ & $0 \cdot 0013$ & $0 \cdot 0008$ & 0 & 0 & 0 \\
\hline $5 \cdot 5$ & $0 \cdot 0026$ & 0.0021 & $0 \cdot 0013$ & $0 \cdot 0008$ & 0 & 0 & 0 & 0 \\
\hline $6 \cdot 0$ & $0 \cdot 0023$ & $0 \cdot 0013$ & $0 \cdot 0008$ & 0 & 0 & 0 & 0 & 0 \\
\hline $6 \cdot 5$ & $0 \cdot 0018$ & $0 \cdot 0009$ & 0 & 0 & 0 & 0 & 0 & 0 \\
\hline $7 \cdot 0$ & $0 \cdot 0009$ & 0 & 0 & 0 & 0 & 0 & 0 & 0 \\
\hline $7 \cdot 5$ & 0 & 0 & 0 & 0 & 0 & 0 & 0 & 0 \\
\hline $8 \cdot 0$ & 0 & 0 & 0 & 0 & 0 & 0 & 0 & 0 \\
\hline $8 \cdot 5$ & 0 & 0 & 0 & 0 & 0 & 0 & 0 & 0 \\
\hline $9 \cdot 0$ & 0 & 0 & 0 & 0 & 0 & 0 & 0 & 0 \\
\hline $9 \cdot 5$ & 0 & 0 & 0 & 0 & 0 & 0 & 0 & 0 \\
\hline $10 \cdot 0$ & 0 & 0 & 0 & 0 & 0 & 0 & 0 & 0 \\
\hline $10 \cdot 5$ & 0 & 0 & 0 & 0 & 0 & 0 & 0 & 0 \\
\hline
\end{tabular}

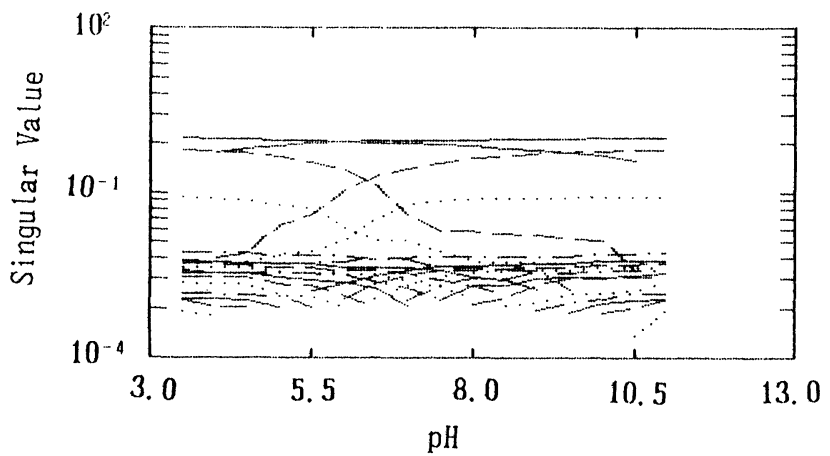

Figure 1. Submatrix analysis of the $C u(I I) / o x /$ en system.

can be distinguished from other minor components, all evolving from the error factors. The error factors are restricted to the lower part of the figure. Cutting the EFA plot at logarithmic singular values lower than $10^{-3}$ yielded clear graph (figure 2), and confirmed that there were only six significant components present. A combined plot of the forward and backward analysis was used to demonstrate where the components arise and where they disappear. Figure 2 demonstrates that new factors, 2, 3, $4,5,6$, appear at around $\mathrm{pHs}$ of $4 \cdot 5,5 \cdot 0,5 \cdot 5,6 \cdot 0$ and $6 \cdot 5$. No further factors were observed until the $\mathrm{pH}$ was 12. The first factor can be related to free

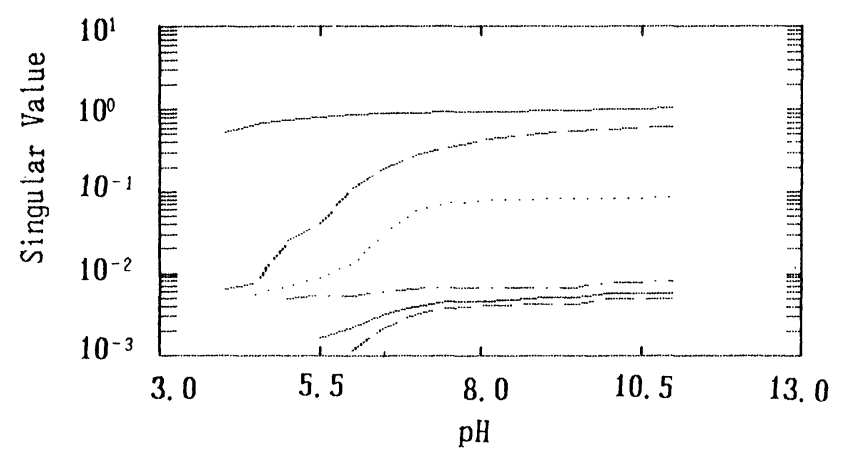

Figure 2. Submatrix analysis of the $\mathrm{Cu}(I I) / 0 x /$ en system for the six species of figure 1 .

$\mathrm{Cu}(\mathrm{II})$ ions because it was present from the beginning of the titration.

Two binary subsystems were investigated by submatrix analysis in the same way. The forward and backward EFA plots showed the appearance and disappearance of a second and a third new factor at around $\mathrm{pH} 4.5$ and 5.0 in the $\mathrm{Cu}(\mathrm{II}) / \mathrm{ox}$ system. These two factors can be attributed to the complex formed between $\mathrm{Cu}(\mathrm{II})$ and oxalate, $[\mathrm{Cu}(\mathrm{ox})]$ and $\left[\mathrm{Cu}(\mathrm{ox})_{2}\right]^{2-}$. Two species had a strong influence upon factors 2 and 3 in the $\mathrm{Cu}(\mathrm{II}) / \mathrm{en}$ 


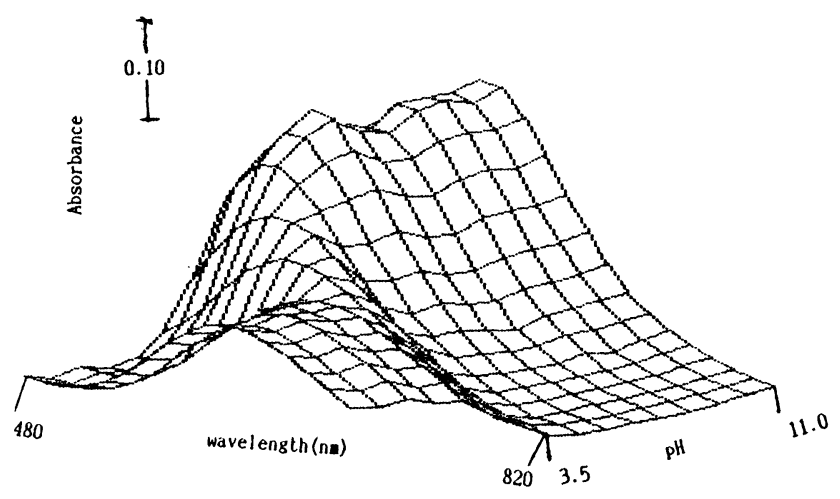

Figure 3. Three-dimensional plot of the spectrophotometric titration data for $\mathrm{Cu}(\mathrm{II}) / \mathrm{ox} / \mathrm{en}$ system.

system; these were two complexes which were formed between $\mathrm{Cu}(\mathrm{II})$ and ethylendiamine, $[\mathrm{Cu}(\mathrm{en})]^{2+}$ and $\left[\mathrm{Cu}(\mathrm{en})_{2}\right]^{2+}$.

Investigation of binary subsystems and chemical reasoning may lead to an association of factors 1, 2, 3, 4, 5 and 6 with the formation of $\mathrm{Cu}(\mathrm{II}), \mathrm{Cu}(\mathrm{ox}),\left[\mathrm{Cu}(\mathrm{ox})_{2}\right]^{2-}$, $\mathrm{Cu}(\mathrm{en})(\mathrm{ox}), \quad[\mathrm{Cu}(\mathrm{en})]^{2+}$ and $\left[\mathrm{Cu}(\mathrm{en})_{2}\right]^{2+}$ in the $\mathrm{Cu}(\mathrm{II}) / \mathrm{ox} / \mathrm{en}$ system. In fact, factor analysis expresses the original data matrix in terms of linear combination of orthogonal vectors. These independent vectors define a space that contains the same information as the data matrix. Associated with each eigenvector is a descriptor of its importance, which is referred to as the eigenvalue. Therefore, all absorbing species have some effect on all the eigenvalues. This was clear in figure 2 at around $\mathrm{pH}$ $5 \cdot 0 .\left[\left(\mathrm{Cu}(\mathrm{Ox})_{2}\right]^{2-}\right.$ was responsible for the introduction of factor 3 and also influenced factor 2. Similarly, at around $\mathrm{pH} 5 \cdot 5, \mathrm{Cu}(\mathrm{en})(\mathrm{ox})$ introduced factor 4 and influenced factors 2 and 3.

Figure 3 shows a three-dimensional plot of the experimental data matrix $D$ obtained in the system of $\mathrm{Cu}(\mathrm{II}) / \mathrm{ox} / \mathrm{en}$. There was no variation of the absorption band due to the $\mathrm{Cu}(\mathrm{II})$ until a $\mathrm{pH}$ value of around $4 \cdot 5-5 \cdot 0$ was reached; this was indicative of the beginning of complexation. In the $\mathrm{pH}$ range where complexation took place, around $4 \cdot 5-11$, the absorption band moved towards lower wavelengths and there was an increase in intensity.

\section{Conclusions}

Evolving factor analysis (EFA) is a powerful method using spectrophotometric titration for the study of speciation of multiple equilibria systems. The method does not require any assumptions about the chemical model of the equilibrium system. Based on mathematical algorithm of EFA, a program called SPGREFA was designed to investigate the speciation of the $\mathrm{Cu}(\mathrm{II}) / \mathrm{ox} / \mathrm{en}$ system. Submatrix analyses are used to estimate the number of species and to show where the species arise and where they disappear. Based on the algorithm of PCA and some newer error functions recommended by Malinowski and others, a program called SPGRAFA was written to estimate the number of species in the system. The results showed that the system contained six species of the type $\mathrm{Cu}(\mathrm{II}),[\mathrm{Cu}(\mathrm{ox})],\left[\mathrm{Cu}(\mathrm{ox})_{2}\right]^{2-},[\mathrm{Cu}($ en $)($ ox $)]$, $[\mathrm{Cu}(\mathrm{en})]^{2+}$ and $\left[\mathrm{Cu}(\mathrm{en})_{2}\right]^{2+}$. This paper demonstrates that the method is an effective way of studying complex systems in environmental samples.

\section{Acknowledgement}

The authors would like to thank the National Natural Science Foundation of China for financial support.

\section{References}

1. Legget, J. (Ed), Computational Methods for the Determination of Formation Constants (Plenum, New York, 1985).

2. Gampp, H., Maeder, M., Meyer, G. J. and Zuberbuhler, A. D., Talanta, 33 (1986), 943.

3. Malinowski, E. R. and Howery, D. E., Factor Analysis in Chemistry (Wiley, New York, 1985).

4. He, X., Li, H. and ShI, H., Fenxi Huaxue, 14 (1986), 34.

5. Malinowski, E. R., Journal of Chemometrics, 1 (1987), 33.

6. Gemperline, P. J. and Hamilton, J. C., In Computer-Enhanced Analytical Spectroscopy, Vol. 2, H. L. C. Meuzelaar, (Ed). (Plenum, New York, 1990). 


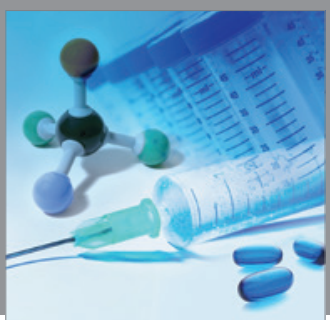

International Journal of

Medicinal Chemistry

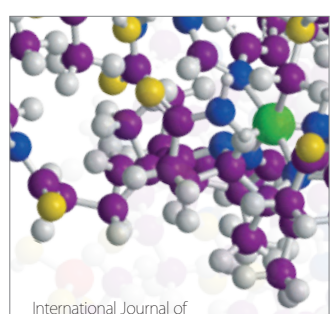

Carbohydrate Chemistry

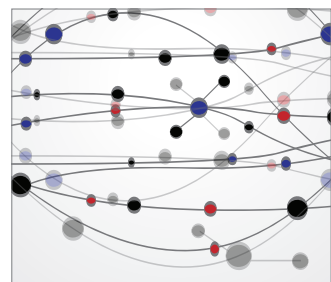

The Scientific World Journal
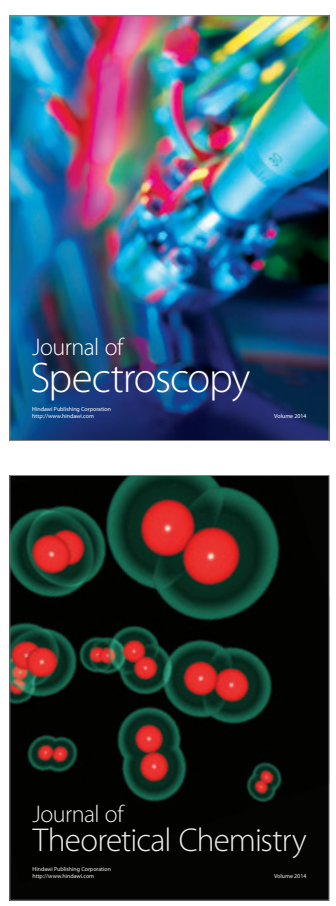
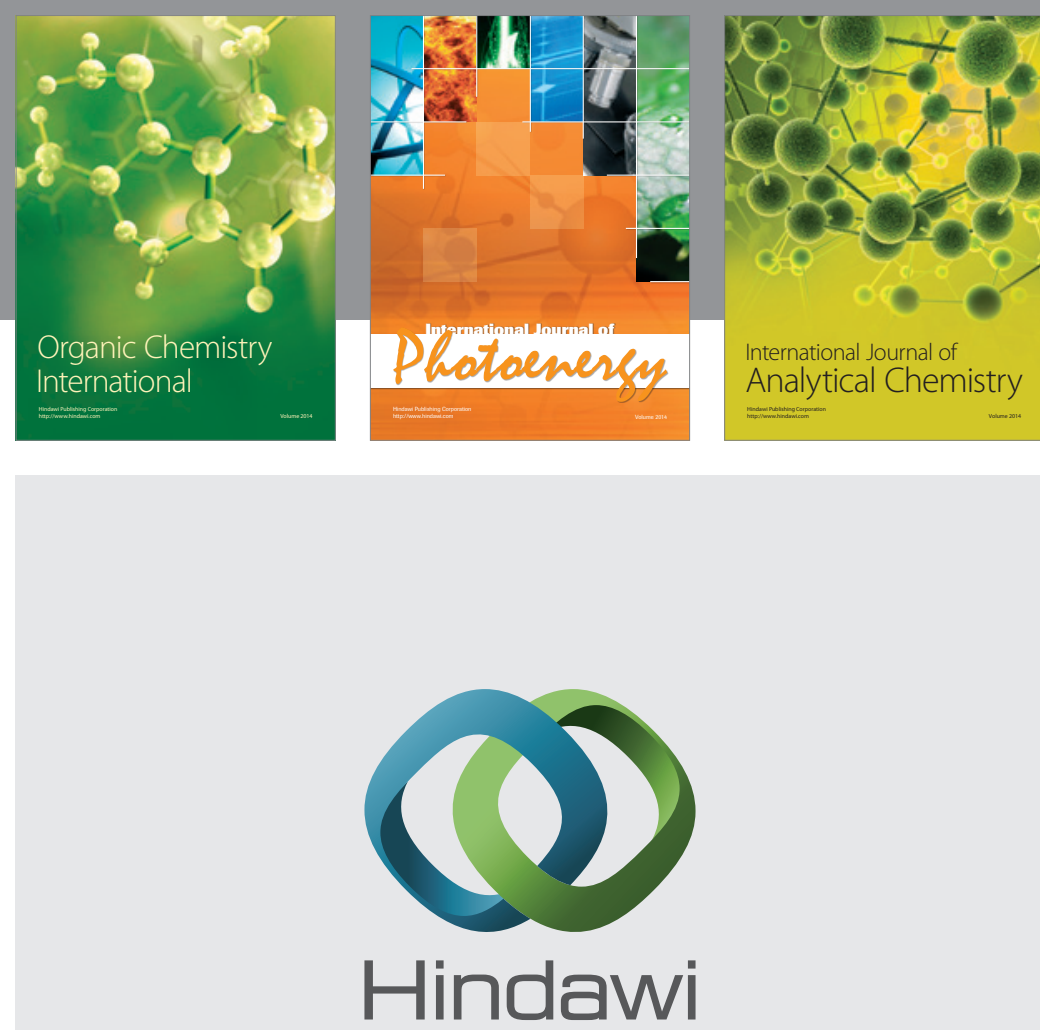

Submit your manuscripts at

http://www.hindawi.com
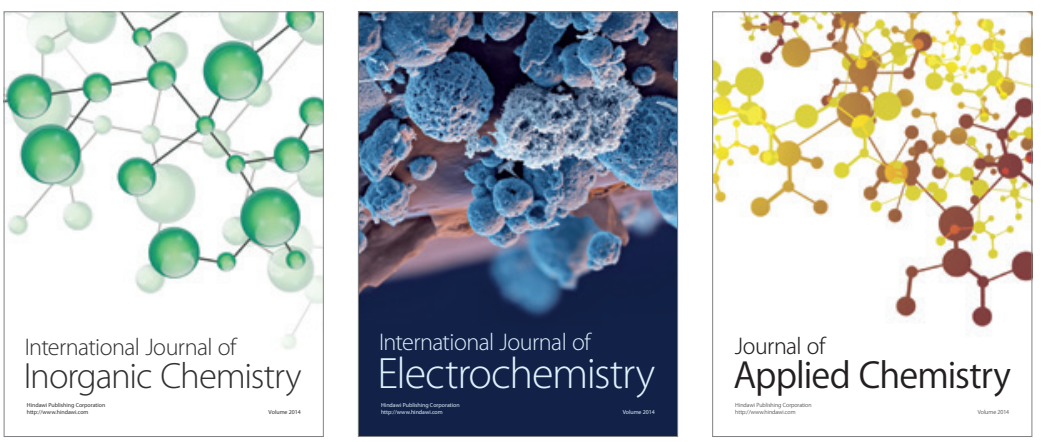

Journal of

Applied Chemistry
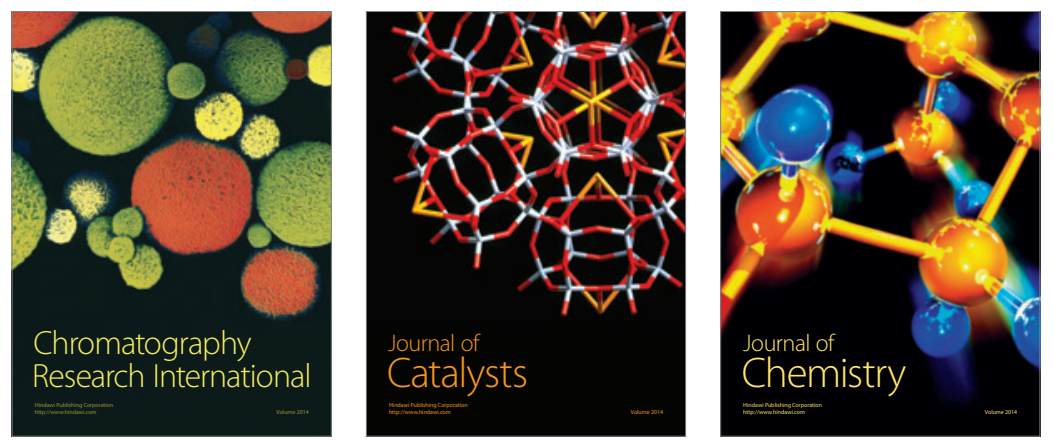
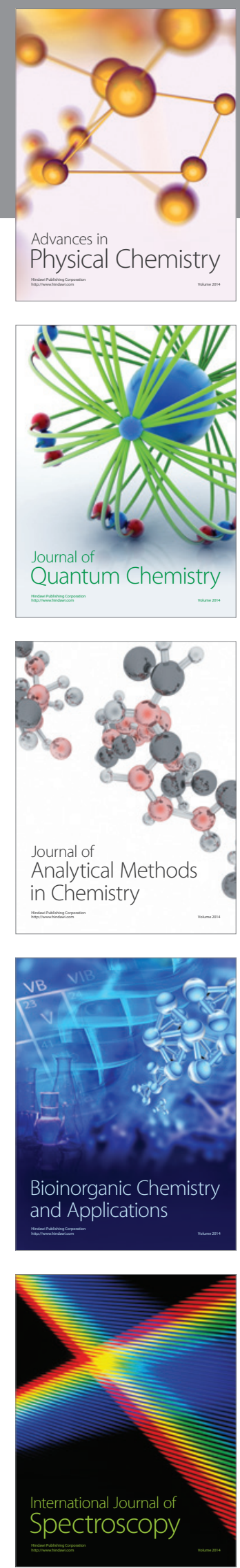